\title{
Main Trends and Perspective SOLUTIONS IN HAPTICS
}

\author{
KharuZin, S. V. \& SHMAKOV, O. A.
}

Abstract: The main objective of this manuscript is to provide comprehensive and highly-structured review of perspective research and commercial solutions in haptics. The subject of presented review is small-and human-sized haptic devices, emerged mainly in past ten years. This paper consists of three main sections. First section provides classification of haptic systems. We consider two classification criteria: device kinematics and the principle of interaction of device's mechanical parts and human-operator. Based on suggested classification four categories of haptic devices is presented: continuous devices, parallel devices, exoskeleton devices and combined devices. In second section a review of research and commercially available haptic devices developed for general and special usage is given. In final section general conclusions are summarized

Key words: Manipulator, haptic feedback, master-slave systems, mechatronics
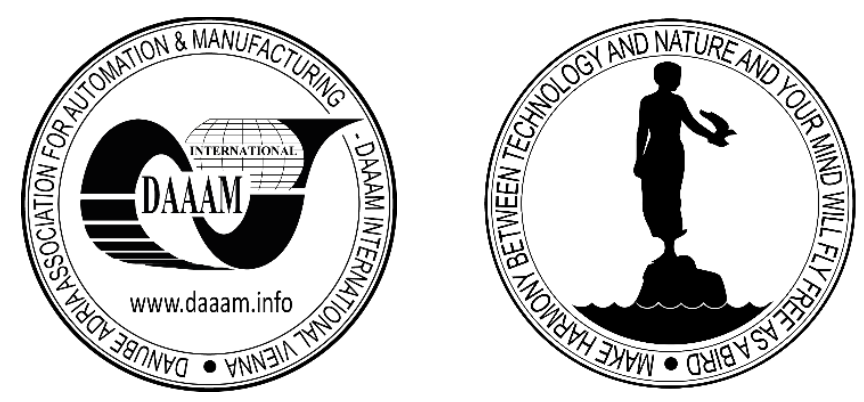

Authors' data: Master at Robotics Kharuzin, S[ergei] V[adimovich]*; Master at Robotics Shmakov, O[leg] A[leksandrovich]*, *Russian State Scientific Center for Robotics and Technical Cybernetics , 21 Tikhoretsky Prospect, Saint Petersburg, 194064, Russia, skharuzin@yandex.ru,shmakov@rtc.ru

This Publication has to be referred as: Kharuzin, S[ergei] \& Shmakov, O[leg] (2017). Main Trends and Perspective Solutions in Haptics, Chapter 02 in DAAAM International Scientific Book 2017, pp.019-028, B. Katalinic (Ed.), Published by DAAAM International, ISBN 978-3-902734-12-9, ISSN 1726-9687, Vienna, Austria DOI: $10.2507 /$ daaam.scibook.2017.02 


\section{Introduction}

The main subject of this paper is haptic devices that can be utilized as a masterpart of control interfaces in tele-operated systems. Development of such devices usually highly correlated with resolving problems belonged to four groups: (1) reducing time delay negative effect (that is inevitable in physical systems), (2) development of mapping algorithms and methods for systems with significant asymmetries (differences in mechanical systems of master and slave devices), (3) establishing of intuitive basis for control process and (4) development of brand new haptic devices for control of wide range of slave devices or for special usage (with specific slave devices).

Wide selection of control devices that are used in tele-operated systems can be divided into two groups. Devices of the first group are joysticks, keypads and etc. Information about changing of condition of some of the control items of such devices converts in forces and torques that than applied to active elements of controlled device. In the simplest case each control item related to one basic function of controlled system. Further sophistication of controlled device's mechanical system may increase the amount of control items. Practical implementation of devices that are overwhelmed by control items can be characterized by excessive cognitive load on user (Cannon D. \& Mel S, 2015). Therefore more developers and researchers utilize haptic devices as a master interfaces in following cases: (1) in case of control of devices with redundant structure, (2) if it is important to establish more obtainable conditions of master interface without excessive sophistication of its structure and (3) if it is impossible or relatively difficult to complete specified task without perceiving scaled proprioceptive and exterioceptive information about controlled device's condition.

The main objective of this manuscript is to provide comprehensive and highlystructured review of the perspective research and commercial solutions in haptics. The subject of presented review is small- and human-sized haptic devices, emerged mainly in past ten years. This paper consists of three main sections. First section provides classification of haptic systems. We consider two classification criteria: device kinematics and the principle of interaction of device's mechanical parts and humanoperator. Based on suggested classification four categories of haptic devices is presented: continuous devices, parallel devices, exoskeleton devices and combined devices. In second section a review of research and commercially available haptic devices developed for general and special usage is given. In final section general conclusions are summarized.

\section{Classification of haptic devices}

Vast majority of existing haptic devices can be divided into four groups: (1) continuous haptic devices, (2) parallel haptic devices, (3) exoskeleton haptic devices and (4) hybrid haptic devices. Devices that belong to 2 first groups consist of fixed base, supporting system and moving plate. Base is used to place device on specially prepared or not prepared surfaces. In most cases developers try to place all active systems in the device's base. Supporting systems consist of one or more link chains 
and playing role of reconfigurable connectors between moving platform (or plate) and fixed base. In most reviewed structures moving platform is directly connected with control handle. Through control handle user feels working environment and estimates remote device's condition.

The way of estimating parameters of interaction between remote device and environment highly depends on amount of so-called control points. Through control points user interacts with remote environment. Current developments can be assigned to two groups: (1) one-point devices and (2) systems with several contact points. In first case user are able to estimate only parameters of interaction of slave device's end point and working environment object. In second case user are able to estimate controlled object's geometrical parameters.

Final classification attribute suggested in scientific literature is device's belonging to impedance or admittance group. Devices of first group generate force feedbacks only when contact with remote environment objects has occurred. Devices of second group generate feedback forces all the time.

\section{Continuous haptic devices}

Continuous haptic devices have only one kinematic chain in their supporting system. In most cases such devices allow user to orient and to position control handle in angular or cylindrical coordinate frame. To reduce negative influence of inertial and gravitational forces applied to moving elements of continuous haptic devices researchers often place all active systems of a device in its base and on the first link of the supporting system. In cases when it is not possible (e. g. when it is important to apply feedback torques to control handle) developers include springs and other passive mechanisms in mechanical structure of haptic devices.

Prototype of haptic device designed to control heavy remote manipulator is described in (Xuewen R. et al., 2014). It consists of 6 continuously combined modules. First five modules belong to supporting system. Last module functions like control handle. Device has relatively large working area. But it's mass highly increased by presence of individual drive in each module. Thereby this leads to increasing of negative inertial forces influence.

Device LHIfAM (LHIfAM, 2017; Borro D. et al., 2004) was developed by Spanish Research Institute CEIT to expand capabilities of staff that performs technical operations with engines of heavy planes. Device's mechanical system consists of continuous manipulator and fixed twin slider system that is used for end effector positioning along two orthogonal axes. Active system of the device provides user with feedback forces applied along three axes.

Haption (Haption, 2017) is French company that brings significant contribution in research in the field of haptics. Haption is affiliated company of CEA (French Nuclear Research Agency) and belongs to European Association for Virtual Reality and French association of Virtual Reality. Company specialized in developing and producing haptic devices in industrial, research and educational areas. Product line of Haption presented by three families of tabletop and large-size haptic devices: Haption Desktop, Haption Virtuoise 6D и Haption Mat 6D (Haption, 2017). 
Devices of Haption Desktop family are presented by two tabletop manipulators: Virtuoise 3D Desktop (Haption, 2017) and Virtuoise 6D Desktop (Haption, 2017). Control handles of both manipulators have three rotational and three positional DOF's. Control handle of Haption Virtuoise 3D Desktop has no any drives. Because of it Haption Virtuoise 6D Desktop is more appropriate for use in teleoperation systems. In spite of Haption Virtuoise 3D Desktop, 6D device has three feedback torque drives. Devices of Haption Virtuoise 6D family are presented by three devices developed especially for teleoperation: Haption Virtuoise 6D Standard (Haption, 2017), Haption Virtuoise 6D TAO (Haption, 2017) and Haption Virtuoise 6D High Force (Haption, 2017). These devices are equiped with more powerful drives in opposite to devices of family Haption Virtuoise 3D Desktop. All six degrees of freedom of such devices are actuated. Device Haption Virtuoise 6D Standard is basic for 6D family. Modifications High Force and TAO have control handles with more sophisticated design and expanded functionality.

Company Geomagic (Geomagic, 2017) specializes on development and producing haptic devices for scientific research, digital sculpting and painting, gaming and etc. Product line of Geomagic presented by two device families: Touch and PHANTOM. All control handles of Touch family devices have 3 actuated DOFs. Devices are highly specified for digital design and sculpting works and therefore their rotational DOFs aren't actuated. Therefore, generated by these devices feedback forces have quite small peak values (Quan-Zen A. et al., 2015). Devices presented in PHANTOM family designed mainly for research in teleoperation area. All 6 DOFs of these devices are actuated.

HapticMaster (FCS Robotics, 2017; Van der Linde R.Q., 2003) is one of the oldest haptic systems presented on market. It is continuous manipulator that performs all working operations in cylindrical coordinate frame. HapticMaster has relatively large value of the exerted feedback force: approximately $250 \mathrm{~N}$.

\section{Parallel haptic devices}

Supporting system of parallel haptic devices consists of several parallel kinematic chains connected from one side with fixed base and from other side with moving platform and control handle. Closed structure of parallel devices determines their relatively high stiffness parameters (Aginaga J. et al., 2011). Such devices provide sufficient redundancy, robustness and capability of working area active reconfiguration. Most of analyzed devices have been built based on the modified deltamechanism scheme. Forward and backward kinematics and dynamics for such systems are given by (Hao J. et al., 2015).

VirtuaPower (Giuk L. et al., 2016) was built as a part of the project for developing cooperative system with several haptic devices and one remote device. Supporting system of this device consists of two 5-link parallel mechanisms, mechanically connected with common base and moving platform. All 3 DOfs of VirtuaPower's control handle are articulated. Device has relatively large size and, therefore, large working area. 
Authors of (Jumpei A. et al., 2011) described a prototype of the parallel device DELTA-R that was built on the base of the modified delta-mechanism scheme. Device's size and dimensions of its working area are almost equal. Basic version of the device consists of fixed base, moving platform and two parallel mechanisms.

Prototype of parallel haptic device and approach for optimizing its structural parameters are described in (Sung-Uk L. \& Seungho K., 2006). Device has three parallel mechanisms in its supporting system that connected with moving platform via spherical joints. All device's drives are placed at the fixed base.

Another haptic device with preliminary optimized parameters presented in (Jung W. Y. et al., 2010). This device designed for interaction with objects of augmented reality. Supporting system of this device consists of thee pantograph mechanisms.

Description of manipulator that is combined from two parallel mechanisms is given by (Minh H. V. \& Uhn J. N., 2010; Vu M. H. \& Uhn J. N. 2011). Every parallel system has three 2-link mechanisms in its structure. Control handle of such device has only 5 DOFs. To achieve one more DOF authors integrated third one-DOF module that is connected to upper parallel mechanism via screw gear.

Haptic system with grasp described in (Lambert P. \& Just H., 2015). All drives of this device placed in the fixed base. Device consists of base, eight legs and integrated grasping mechanism for user fingers. Base of the device has form of a box. On each edge of the box two parallel 2-linked mechanisms are placed.

Device (Dongseok R., 2009) is a combination of two parallel mechanisms. It is designed as part of control interface for mobile robots with onboard manipulators. Lower parallel mechanism consists of fixed base and three 2-link continuous mechanisms. Rotation axes of all lower mechanism joints are parallel. Upper mechanism developed on the base of modified delta-mechanism and has one positional and three rotational DOFs.

Haptic device with three grasps for user fingers described in (Huanhuan Q. et al., 2015). All grasps are independently actuated. Independent actuation is needed to establish three-point interaction with remote environment. Fixed base consists of three consequently inserted in each other aluminum profiles. On the top of upper profile holder for supporting system is mounted. Structure of the device allows user to reconfigure working area without interruption of teleoperation process.

Novint Falcon is a haptic device developed by Novint (Novint Inc., 2017) company for use in scientific research, digital sculpting and gaming. Axes of Falcon delta-mechanism oriented collinearly to sides of triangle placed in the center of its base. Device has relatively small dimensions and values of reflected forces.

Force Dimension (Force Dimension, 2017) is one of the leaders in developing and production high precision interfaces for remote surgery. All produced by company devices are modified delta mechanisms. Product line of haptic devices consists of three device families (Force Dimension, 2017): Delta.X, Omega.X и Sigma.X. Devices differ in dimensions and functionality of control handles.

Quanser (Quanser , 2017) is one of the main world producers of research and study haptic solutions. It provides production for approximately 2,500 educational institutions. Quanser haptic devices model line presented by three products (Quanser, 2017). First device is Twin-Pantograph 3-DOF Haptic Interface. It consists of two 
parallel pantograph mechanisms connected by common rigid link. It has layered flat structure and therefore provides only three DOFs for positioning and orienting control handle. Supporting system of 5-DOF Haptic Wand also consists of two pantographs that attached to fixed base via pairs of active joints with orthogonal rotation axis. Because of it control handle has two orientation and three positional degrees of freedom. Most sophisticated Quanser haptic device is $\mathrm{HD}^{2}$ High Definition Haptic Device. It consists of two continuous manipulators placed in parallel. Each manipulator has 3 DOFs. Both manipulators are rigidly attached to fixed base and their output links are mechanically connected via handle.

\section{Exoskeletal haptic devices}

Sensors and drives of the exoskeletal haptic devices are placed directly on user. These devices do not establish functional strengthening of human-operator and therefore have relatively low mass and small packed size.

Kento (Kazuo H. et al., 2009) have been developed at University of Tokyo. Concerned haptic device can be characterized as a wearable actuated suit for upper half of user's body. Device has been designed as a control interface for anthropomorphous robot Kojiro (Ikuo M. et al., 2007). To transfer forces and torques from remote environments authors suggest using so called muscle elements as drives. Muscle element consists of enclosed frame, drive, cable and spring. Drive regulates spring stiffness to form variable mechanical resistance for user movements.

Exoskeletal system with tactile feedback described in (Yosuke M. et al., 2015). System has been designed for teleoperation with anthropomorphous robot. It consists of augmented reality helmet, tactile gloves and backpack with control computer and batteries. All components connected via so called sensor tubes. Orientation and position of user hands and head are estimated based on sensor tube configuration. Each tube is a set of several rigid links. Mutual position of these links is determined by potentiometers placed between them.

Synergistical approach for developing of exoskeletal haptic glove for control of systems with significant asymmetries suggested in (Brygo A. et al., 2016). Glove has three actuated fingers. Each finger consists of 6 links connected by 5 passive and 1 active joints. Feedback forces are produced via applying torques to the first link of each finger.

Company CyberGlove Systems (CyberGlove Systems, 2017) specializes on development of exoskeletal and hybrid haptic systems with tactile and force feedback. Model line of CyberGlove Systems presented by exoskeletal CyberGlove, CyberGrasp and hybrid CyberForce devices. CyberGlove designed as compliant glove that provides tactile feedback. It includes up to 22 resistive strain sensors that provides devices control system with information about user hand configuration. CyberGrasp is a combination of CyberGlove and rigid exoskeleton glove with force feedback. Rigid exoskeleton consists of 5 similar mechanisms with adjustable geometry. Each mechanism actuated by independent cable drive. CyberForce is a combination of CyberGrasp and six-DOF continuous manipulator. Detailed analysis of kinematics and dynamics for such device was performed in (Martrinez-Terran G. et al., 2015). 


\section{Haptic devices with hybrid structure}

Hybrid manipulators are a class of devices that combine features of continuous, parallel and exoskeletal systems.

iFeel3 (Chaobin L. et al., 2010) was designed for interaction with objects of augmented reality. Mechanical system of the device consists of fixed base, pantograph and control handle. Pantograph is connected with fixed base via two active joints with orthogonal axes of rotation. Parallel mechanism elements establish passive compensation of gravitational forces. Device has relatively small overall dimensions and small operational area.

Haptic system (Hongmin W. et al., 2010) consists of a parallel platform and active control handle that is designed as continuous mechanism. Device is operated as high precision interface for control of medical manipulators. Parallel part of supporting system is a modified delta-mechanism. Control handle performs working operations in angular coordinate frame.

Compact modular haptic device presented in (Uchiyama M. et al., 2007). It has combined continuous-parallel structure. Device consists of a parallel mechanism, twoDOF active universal mechanism and one-DOF mechanism that is mechanically combined with control handle.

\section{Conclusion}

A brief review of recently developed research and commercially available mastersolutions for teleoperation was given. Considered devices were classified in four groups by their kinematic structure and principles of mechanical interaction with user. Distinctive features along with benefits and disadvantages of these groups were analyzed.

Based on performed analysis a few conclusions are made as follows. Continuous haptic devices have relatively simple kinematics and small size of active subsystems. However, increasing target stiffness of such structures increases their dimensions too. More stiff and relatively small devices are parallel mechanisms. Nevertheless, such systems have complex kinematics and their implementation in teleoperation interfaces related with utilization of physical to virtual working space mapping methods. Exoskeletal haptic devices provide sufficient intuitive basis for interaction between user and remote environment objects. Hybrid systems combine benefits and disadvantages of all featured groups.

\section{References}

Cannon D. \& Mel S. (2015). Perceived mental workload and operator performance of dexterous manipulators under time delay with master-slave interfaces, Proceedings of 2015 IEEE International Conference on Computational Intelligence and Virtual Environments for Measurement Systems and Applications (CIVEMSA), ISBN 978-14799-6092-7, Shenzhen, China, June 2015, Published by IEEE 
Xuewen R.; Bin L.; Hui C. \& Guoqing C. (2014). Design of a Force Feedback Teleoperation Master Manipulator, Proceedings of the 2014 IEEE International Conference on Information and Automation, ISBN 978-1-4799-4100-1, Hailar, China, October 2014, Published by IEEE

*** http://www4.tecnun.es/asignaturas/control1/LHIfAM.pdf - Electronic source with LHIfAM specification, LHIfAM (2017), Accessed on: 04-10-2016

Borro D.; Savall J. \& Amundarain A. (2004). A Large Haptic Device for Aircraft Engine Manipulability. IEEE Computer Graphics and Applications, Vol. 24, No. 6 (Nov.-Dec. 2004) pp. 70 - 74, ISSN 0272-1716

*** http://www.haption.com/site/index.php/en/company-profile-menu-en - Official site of Haption, Haption (2017), Accessed on: 2017-04-10

*** http://www.haption.com/site/pdf/Datasheet_Mat_6D.pdf - Electronic source MAT-6D specification, Haption (2017), Accessed on: 2017-04-10

*** http://www.haption.com/site/pdf/Datasheet_Virtuose_3DDesktop.pdf Electronic source with Virtuoise 3D Desktop specification, Haption (2017), Accessed on: 2017-04-10

*** http://www.haption.com/site/pdf/Datasheet_Virtuose_6DDesktop.pdf Electronic source with Virtuoise 6D Desktop specification, Haption (2017), Accessed on: $2017-04-10$

*** http://www.haption.com/site/pdf/Datasheet_Virtuose_6D_Standard.pdf Electronic source with Virtuoise 6D Standard specification, Haption (2017), Accessed on: 2017-04-10

*** http://www.haption.com/site/pdf/Datasheet_Virtuose_6D_TAO.pdf - Electronic source with Virtuoise 6D TAO specification, Haption (2017), Accessed on: 2017-0410

*** http://www.haption.com/site/pdf/Datasheet_Virtuose_6D_HF.pdf - Electronic source with Virtuoise 6D High Force specification, Haption (2017), Accessed on: 2017-04-10

*** http://www.geomagic.com/en/products-landing-pages/haptic - Official site of Geomagic, Geomagic (2017), Accessed on: 2017-04-10

Quan-Zen A.; Ben H. \& Saeid N. (2015). Multipoint Haptic Mediator Interface for Robotic Teleoperation. IEEE Systems Journal, Vol. 9, No. 1, (March 2015), pp. 86-97, ISSN 1932-8184

*** http://www.vrlab.ctw.utwente.nl/eq/Documentation/HapticMaster_HM_Product Sheet\%20(final).pdf - Electronic source with Haptic Master specification, FCS Robotics (2017), Accessed on: 2017-04-10

*** https://people.cs.vt.edu/ wangr06/touch\%20review\%20origanization/

VanLFR02.pdf-The HapticMaster, a new high-performance haptic interface, Van der Linde R.Q. (2002), Accessed on: 2017-04-10

Aginaga, J.; Altuzarra, O.; Iriarte, X. \& Macho, E. (2011). Stiffness of Parallel Manipulators with Crank-Connecting Rod System, Chapter 38 in: DAAAM International Scientific Book 2011, B. Katalinic (Ed.), pp. 465-476, Published by DAAAM International, ISBN 978-3-901509-84-1, Vienna, Austria

Hao J.; Bian G.; Xie X.; Hou Z. \& Yu H. (2015). Kinematic and Static Analysis of a Cable-driven 3-DOF Delta Parallel Mechanism for Haptic Manipulators, Proceedings 
of the 34th Chinese Control Conference, ISBN 978-9-8815-6389-7, Hangzhou, China, July 2015, Published by IEEE

Giuk L.; Sung-moon H. \& Yonghwan O. (2016). A Novel Haptic device with Highforce Display Capability and Wide Workspace, Proceedings of the 2016 IEEE International Conference on Robotics and Automation (ICRA), ISBN 978-1-46738026-3, Stockholm, Sweden, May 2016, Published by IEEE

Jumpei A., Hiroyuki K. \& Norio I. (2011). Haptic Device Using a Newly Developed Redundant Parallel Mechanism. IEEE Transactions On Robotics, Vol. 27, No. 2, (April 2011) pp. 201-214, ISSN 1552-3098

Sung-Uk L. \& Seungho K. (2006). Analysis and Optimal Design of a New 6 DOF Parallel Type Haptic Device, Proceedings of the 2006 IEEE/RSJ International Conference on Intelligent Robots and Systems, ISBN 1-4244-0258-1, Beijing, China, October 2006, Published by IEEE

Jung W. Y.; Jeha R. \& Yoon-Know H. (2010). Optimum design of 6-DOF parallel manipulator with translational/rotational workspaces for haptic device application. Journal of Mechanical Science and Technology, Vol. 31, No. 260, (February 2010) pp. 1151-1162, ISSN 1976-3824

Minh H. V. \& Uhn J. N. (2011). A New 6-DOF Haptic Device for Teleoperation of 6DOF Serial Robots. IEEE Transactions On Instrumentation And Measurement, Vol. 60, No. 11, (Nov. 2011) pp. 3510-3523, ISSN 1557-9662

Vu M. H. \& Uhn J. N. (2010). Tele-operation of a 6-DOF Serial Robot Using a New 6-DOF Haptic Interface, Haptic Audio-Visual Environments and Games (HAVE), Proceedings of the 2010 IEEE International Symposium, ISBN 978-1-4244-6509-5, Phoenix, AZ, USA, USA, October 2010, Published by IEEE

Lambert P. \& Just H. (2015). A Novel Parallel Haptic Device with 7 Degrees of Freedom, Proceedings of the 2015 IEEE World Haptics Conference (WHC), Northwestern University, ISBN 978-1-4799-6624-0, Evanston, Il, USA, June 2015, Published by IEEE

*** http://www.sciencedirect.com/science/article/pii/S0957415809001895

Development of a six DOF haptic master for teleoperation of a mobile manipulator, Dongseok R. (2009), Accessed on: 2017-04-10

Huanhuan Q.; Aiguo S.; Yuqing L.; Guohua J. \& Bohe Z. (2015). Design and Calibration of a New 6 DOF Haptic Device. Sensors, Vol. 15, No. 12, (Dec. 2015), pp. 31293-31313, ISSN 1424-8220

*** http://www.novint.com/index.php/novintfalcon - Official site of Novint, Novint Inc. (2017), Accessed on: 2017-04-10

*** http://www.forcedimension.com/company/about - Official site of Force Dimension, Force Dimension (2017), Accessed on: 2017-04-10

*** http://www.forcedimension.com/products - Electronic resource with Force Dimension product specifications, Force Dimension (2017), Accessed on: 2017-04-10 *** http://www.quanser.com - Official site of Quanser, Quanser (2017), Accessed on: 2017-04-10

*** http://www.quanser.com/Products/Docs/74/5DOF_Haptic_Wand.pdf Electronic source with 5-DOF Haptic Wand specification, $\bar{Q}$ uanser (2017), Accessed on: $2017-04-10$ 
Kazuo H.; Mariko Y.; Yuto N.; Ikuo M. \& Masayuki I. (2009). Development of Bilateral Wearable Device "Kento" for Control Robots using Muscle Actuator Modules, Proceedings of the 18th IEEE International Symposium on Robot and Human Interactive Communication, ISSN 1944-9445, Toyama, Japan, September 2009, Published by IEEE

Ikuo M.; Yuto N.; Yoshinao S.; Yuta N.; Tamaki N.; Naoya M.; Juinichi U.; Kazuo H.; Tomoaki Y. \& Massayuki I. (2007). An Advanced Musculoskeletal Humanoid Kojiro, Proceedings of the 2007 IEEE-RAS International Conference on Humanoid Robotics, ISBN 978-1-4244-1861-9, Pittsburgh, PA, USA, April 2009, Published by IEEE

Yosuke M. ; Akio N. \& Kenta N. (2015). Operation Assist for a Teleoperated Robot System Controlled with a Lightweight and High-Operable Master Device, Proceedings of the 2015 IEEE/SICE International Symposium on System Integration (SII), Meijo University, ISBN 978-1-4673-7242-8, Nagoya, Japan, December 2015, Published by IEEE

Brygo A., Sarakoglou I.; Ajoudani A.; Hernandez N. G.; Grioli G.; Catalano M.; Caldwell D. G. \& Tsagarakis N. (2016). Synergy-Based Interface for Bilateral Telemanipulations of a Master-Slave System with Large Asymmetries, Proceedings of the 2016 IEEE International Conference on Robotics and Automation (ICRA), ISBN 9781-4673-8026-3, Stockholm, Sweden, May 2016, Published by IEEE

*** http://www.cyberglovesystems.com - Official site of CyberGlove Systems, CyberGlove Systems (2017), Accessed on: 2017-04-10

Martrınez-Terran G.; Omar A. D.-R.; Juan D. R.-Z.; Luis E. R.-V.; Ismaylia S.-U. \& Alejandro L.-V. (2015). CyberForce Haptic Device: Kinematics and Manipulability, Proceedings of the 2015 International Conference on Mechatronics, Electronics and Automotive Engineering, ISBN 978-1-4673-8329-5, Prague, Czech Republic, January 2016, Published by IEEE

Chaobin L.; Dangxiao W. \& Yuru Z. (2010). iFeel3: a haptic device for virtual reality dental surgery simulation, Proceedings of the International Conference on Virtual Reality and Visualization, ISBN 978-1-4577-2156-4, Beijing, China, Published by IEEE

Hongmin W.; Dongmei W.; Zhijiang D.; Zhiyhuan Y. \& Changjun L. (2010). Design of a Novel Serial and Parallel Force Feedback Master Manipulator, Proceedings of the 2010 IEEE International Conference on Robotics and Biomimetics, ISBN 978-1-42449318-0, Tianjin, China, December 2010, Published by IEEE

Uchiyama M., Tsumaki Y., Yoon W.-K. (2007). Design of a compact 6-dof haptic device to use parallel mechanisms. Springer Tracts in Advanced Robotics, vol 28., pp 145-162, ISSN 1976-3824 